\title{
Alcohol and depression in military-related traumatic stress
}

\author{
Aloui S, Lansari R, Krir MW, El Kefi H, Oumaya A \\ Psychiatry department, Military Hospital of Tunis, Tunisia
}

\section{Introduction:}

Mental health concerns such as depression and PTSD are significant issues among the military population and both conditions have been linked to higher levels of alcohol use. Military-related traumatic stress seems to elevate risk for individuals to misuse alcohol. Turning to alcohol consumption may be a way to numb their negative feelings and pain. Comorbid depression appears to worsen rates of alcohol use in military members with post traumatic stress disorder (PTSD).

\section{Objective:}

A cross-sectional correlational study of a sample of 60 military members treated for PTSD in the principal military hospital was conducted to evaluate the prevalence of alcohol use and the role of comorbid depression as a risk factor.

\section{Methods:}

All patients completed the following self-administered scales:

- The PTSD Checklist for DSM-5 (PCL-5) to assess the 20 DSM-5 symptoms for PTSD

- The Beck depression inventory (BDI) to assess the symptoms of depression

- Alcohol use Identification Test - Consumption questionnaire (AUDIT-C) to assess alcohol use

\section{Results:}

All participants were male. The mean age of the sample was 32, 85 years.

The majority of the patients were under 30 years, non commissioned officers, single and with no personal history of psychiatric illness.

PCL-5 scores ranged from 0 to 68 with a mean of 34,89 . Severe PTSD (PCL5>33) was found in $54,2 \%$ of the patients.

BDI scores ranged from 0 to 36 with a mean of 14,05 .

Depression was associated with PTSD in $79,7 \%$ of the cases.

PTSD patients turned to alcohol use in $23,7 \%$ of cases.

BDI scores were positively correlated with total PCL5 scores $(r=0,892, p<0,001)$.

Alcohol consumption was positively correlated with the presence of comorbid depression $(\mathbf{p}=\mathbf{0 , 0 1 9})$ and the severity of PTSD $(p=0,026)$. correlations table

\begin{tabular}{|c|c|c|}
\hline P & Depression & PCL 5 T \\
\hline Alcohol use & 0.019 & 0.026 \\
\hline
\end{tabular}

\section{Discussion:}

Results in this study suggest that alcohol consumption is related to depression and PTSD symptoms in Tunisian military patients.

Rates of alcohol misuse in the UK Armed Forces are higher than the general public (Henderson et al 2009; Hooper et al., 2008). In a cohort study of nearly 10,000 military personnel, $13 \%$ reported alcohol misuse (Head et al., 2016).

The same study reported that $45 \%$ of military personnel with probable PTSD had a co-occurring problem with alcohol misuse.

Other studies suggest that depression or PTSD symptoms may not be related to drinking level, but may predict levels of alcohol problems. (Dennhardt and Murphy, 2011; McDevitt-Murphy et al., 2010).

Evidence suggests that having problems with alcohol is associated with poorer long-term PTSD treatment response (Murphy, Palmer, Westwood, Busuttil, \& Greenberg, 2016).

Additionally, depression and PTSD have been found to predict poor response to alcohol interventions, perhaps due to the fact that alcohol misuse among persons with depression or PTSD often serves as a maladaptive coping strategy (Geisner et al., 2007; Hien et al., 2000; Kaysen et al., 2007)

Alcohol misuse can also have an impact on treatment utilisation, with treatment-seeking veterans with alcohol problems attending fewer mental health visits and being more likely to have a negative perception of mental health treatment when alcohol misuse is comorbid with depression or PTSD (Elbogen, Wagner et al., 2013).

\section{Conclusion:}

A depressed individual, who developed PTSD, is highly more likely to use alcohol particularly following exposure to military-related trauma. Additional consideration of other variables would also be valuable. Results of such studies could have important implications for understanding comorbidity of PTSD and substance use disorders. 